\title{
MODELLING THE CONODONT SKELETON: A NEW RECONSTRUCTION FOR THE CONODONT MOUTH
}

DONOGHUE*, Philip C. J. \& PURNELL, Mark A., Department of Geology, University of Leicester, University Road, Leicester LE1 7RH, U.K.

The mode of operation and precise function of the conodont apparatus has been a subject of long standing controversy. This stems from an absence of detailed knowledge of the spatial arrangement of the component elements of the apparatus, with the result that many hypotheses of function have been rather poorly constrained and, in some cases, completely without foundation. The paucity of detail regarding conodont skeletal architecture reflects the nature of the conodont fossil record; almost all conodonts are recovered by acid dissolution of limestones and disaggregation of shales and these isolated elements preserve no information about their original orientation or arrangement in the living animal. Very rarely, however, conodont animals died in environments conducive to the preservation of primary spatial information. So-called 'bedding plane assemblages' represent such occurrences, where a lack of scavenging and current activity, associated with dysaerobic or anaerobic bottom-waters, conspired to preserve the elements in the position they attained after death and decay of the animal's soft tissues. Bedding plane assemblages thus represent passive collapse into two-dimensions of the threedimensional skeletal apparatus. Depending on the orientation of the carcass relative to the sea bed, the pattern of elements in the bedding plane assemblage will vary, with lateral collapse (i.e. carcass lying on its side), dorso-ventral collapse, and anterior collapse producing very different final arrangements of elements. These different arrangements are analogous to views of the apparatus from the side, above, or from the front. One view is not enough to reconstruct a three-dimensional structure, but analysis of several hundred bedding plane assemblages has allowed us to recognise a limited number of recurrent collapse patterns or views.

Reconstructing the conodont apparatus requires that we reverse the process of collapse by building a physical model of the skeleton. The three dimensional arrangement of the model can be tested by simulating collapse of the apparatus using two dimensional projections, produced photographically. If the variety of different bedding plane assemblage patterns of collapse can be matched by photographs, then the reconstructed architecture must be correct. We have used this technique to produce the first precise scale model of the ozarkodinid apparatus. This indicates that the apparatus comprised an anterior array of nine closely spaced $\mathrm{S}$ elements, arranged as two oblique, bilaterally opposed sets of four elements $\left(\mathrm{Sb}_{1}, \mathrm{Sb}_{2}, \mathrm{Sc}_{1}, \mathrm{Sc}_{2}\right)$ stacked on either side of an axial, symmetrical Sa element. The $\mathrm{S}$ elements were flanked by a pair of pick-shaped $\mathrm{M}$ elements, the long axes of which were not parallel to the $S$ elements. Two opposed pairs of P elements lay at the posterior of the apparatus.

Our new three dimensional reconstruction of ozarkodinid skeletal architecture imposes a number of constraints on hypotheses of apparatus function and conodont feeding. The arrangement of $\mathrm{S}$ and $\mathrm{M}$ elements indicates grasping of food could be achieved by a simple anterior-posterior motion of the apparatus accompanied by bilateral opening and closure, analogous to the operation of the lingual teeth of hagfish. Crucially, the model has also allowed us to recognise the interactive functional surfaces of opposed pairs of P elements; surfaces which are now known to show wear patterns resulting from the slicing and crushing of food. 